\title{
Cost-effectiveness of strontium ranelate in the treatment of male osteoporosis
}

\author{
M. Hiligsmann • W. Ben Sedrine • O. Bruyère • \\ J.-Y. Reginster
}

Received: 14 September 2012 / Accepted: 8 January 2013 /Published online: 1 February 2013

(C) The Author(s) 2013. This article is published with open access at Springerlink.com

\begin{abstract}
Summary The results of this study suggest that, under the assumption of same relative risk reduction of fractures in men as for women, strontium ranelate could be considered a cost-effective strategy compared with no treatment for the treatment of osteoporotic men from a Belgian healthcare payer perspective.

Introduction This study was conducted to estimate the costeffectiveness of strontium ranelate in the treatment of osteoporotic men.

Methods A previously validated Markov microsimulation model was adapted to estimate the cost $(€ 2,010)$ per quality-adjusted life-year (QALY) gained of strontium ranelate compared with no treatment. Similar efficacy data on lumbar spine and femoral neck bone mineral density (BMD) between men with osteoporosis at high risk of fracture (MALEO Trial) and postmenopausal osteoporotic women (pivotal SOTI, TROPOS trials) supports the assumption, in the base-case analysis, of the same relative risk reduction of fractures in men as for women. Analyses were conducted, from a Belgian healthcare payer perspective, in the population from the MALEO Trial who is a men population with a mean age of 73 years, and BMD T-score $\leq-2.5$ or prevalent vertebral fracture (PVF).
\end{abstract}

M. Hiligsmann

Department of Health Services Research, School for Public Health and Primary Care (CAPHRI), Maastricht University, Maastricht, The Netherlands

M. Hiligsmann $\cdot$ W. Ben Sedrine $\cdot$ O. Bruyère $\cdot$ J.-Y. Reginster Department of Public Health, Epidemiology and Health Economics, University of Liège, Liège, Belgium

M. Hiligsmann $(\square)$

Department of Health Services Research, Maastricht University, P.O. Box 616, 6200 MD Maastricht, The Netherlands

e-mail: m.hiligsmann@maastrichtuniversity.nl
Results In the MALEO population, strontium ranelate compared with no treatment was estimated at $€ 49,798$ and $€ 25,584$ per QALY gained using efficacy data from the intent-to-treat analysis and the per-protocol analysis including only adherent patients, respectively. In men with a BMD T-score $\leq-2.5$ or with PVF, the cost per QALY gained of strontium ranelate fall below thresholds of $€ 45,000$ and $€ 25,000$ per QALY gained based on efficacy data from the entire population of the clinical trial and from the perprotocol analyses, respectively.

Conclusions The results of this study suggest that, under the assumption of same relative risk reduction of fractures in men as for women, strontium ranelate could be considered cost-effective compared with no treatment for male osteoporosis.

Keywords Cost-effectiveness $\cdot$ Fractures $\cdot$ Men · Osteoporosis $\cdot$ Strontium ranelate

\section{Introduction}

Osteoporosis in men is increasingly recognized as a major public health problem [1]. Although osteoporosis is less common in men than in women, it has been estimated that around $30 \%$ of hip fractures occur in males and one out of five men aged 60 years will experience an osteoporotic fracture during their remaining lifetime [2, 3]. In Belgium, the female/male ratio of hip fractures significantly decreased from 3.12 to 2.97 between 2000 and 2007 and is expected to further decrease to 2.52 by the year 2025 [2]. With increasing life expectancies in men and higher excess mortality after hip fractures in men than in women [4], osteoporosis in men will become a large burden on society and healthcare systems.

Current treatments available for male osteoporosis, however, remain limited including alendronate, risedronate, 
zoledronate and parathormone [1]. Strontium ranelate has been primarily developed and approved for the treatment of postmenopausal osteoporosis. In clinical trials in postmenopausal women with osteoporosis, strontium ranelate has been shown to be safe and effective in reducing the risk of vertebral and non-vertebral fractures in a wide scatter of patients, from osteopenia to very elderly subjects, over a long period (up to 10 years) [5-9]. The cost-effectiveness of strontium ranelate in postmenopausal women has also been demonstrated in different settings [10-14].

Recently, strontium ranelate also demonstrated to be effective for the treatment of male osteoporosis in a multicentre randomised controlled trial (i.e., the MALEO Trial) [15]. Under continuing economic pressure, the assessment of a new health intervention, however, goes beyond the three regulatory criteria of quality, safety and efficacy. The assessment of cost-effectiveness is considered as the fourth hurdle to market, and plays an increasingly role in healthcare decision making [16]. Many countries have introduced formal requirements for economic analyses as part of the pricing or reimbursement decisions [17]. As the economic value of strontium ranelate in populations of men has not been analysed yet, this study aims to estimate the costeffectiveness of strontium ranelate, compared with no treatment, for the treatment of Belgian men with osteoporosis or a prevalent vertebral fracture (PVF).

\section{Materials and methods}

\section{Economic model}

The simulation model is the same as the model developed for postmenopausal osteoporotic (PMO) women which has been validated [18] and used in many published health economic analyses [12, 13, 19-23]. Recently, an updated version of the model using a 6-monthcycle length has been developed [23]. This last model version was slightly revised in this study to also include a health state for venous thromboembolic events (VTEs). The model was programmed using the software TreeAge Pro 2011 (TreeAge Pro Inc., Williamston, MA, USA).

The Markov model health states are no fracture, death, VTE, hip fracture, clinical vertebral fracture, wrist fracture, other fracture and the corresponding post-fracture states. Post-fracture states were created as some parameters (e.g., fracture disutility) were estimated over a 1-year period [23]. All patients, one at a time, began in the 'no fracture' state and had, every 6 months, a probability of having a fracture at the hip, clinical vertebrae, wrist, or other site or of dying. Patients in a fracture state can stay in the same fracture state if they re-fracture, change to another fracture state, die or change in the next cycle to the post-fracture state. Because hip fracture is associated with extra costs in the year following the fracture that are greater than the hospitalization cost of any other fractures, patients who have had a hip fracture were only at risk for another hip fracture or dying in the first cycle following the fracture. Patients being in any postfracture state might have a new fracture (all fracture types are possible), die or move to the 'no fracture' state. The probability for patients to move to the VTE health state was also considered under treatment with strontium ranelate.

Fracture data

A description of the different components of the model is provided below. Model data are included in Table 1. Readers are also referred to previously published research for further details and limitations of the model [17].

The incidence of hip fractures in the general men population was derived from the national database of hospital bills (average of the years 2005-2007) [2]. Since the incidence of other fractures was not known, we assumed that the age-specific ratio of index fracture to hip fracture in Belgium was the same as found in Sweden [3]. This assumption, used in the development of many FRAX ${ }^{\circledR}$ models including Belgium [24], appears to be appropriate for West European countries, the USA and Australia [25].

Age-specific mortality rates were obtained from the National Institute of Statistics. According to data from a recent meta-analysis [4], hip fractures increased male death probabilities by 5.75 in the first 6 months following the fracture, by 2.315 in the period 6-12 months and by 1.691 in subsequent years. As the increased mortality following clinical vertebral fractures has been found in many studies to be very similar than those of a hip fracture [26-29], the same impact was assumed after hip and clinical vertebral fractures. To avoid an overestimation of the beneficial effect of treatment on mortality, it is important to take only into account excess mortality that are directly or indirectly attributable to the fractures themselves [30], which could be reduced through fracture prevention. Because excess mortality may also be attributable to comorbidities, we assumed in the model only $25 \%$ of the excess mortality after fractures $[28,31]$.

A healthcare payer perspective including direct medical costs was adopted for all cost estimates, as recommended for pharmacoeconomic evaluations in Belgium [32]. Following the guideline, direct healthcare costs paid by the national health insurance and patient's out-of pocket costs were included [32]. All costs were expressed in the year 2010 using the healthcare product price index when necessary, and discount rates of $3 \%$ for costs and of $1.5 \%$ for health benefits were assumed for the base-case analysis also based on the Belgian guideline for pharmacoeconomic evaluations [32]. The direct hospitalisation cost of hip fracture, 


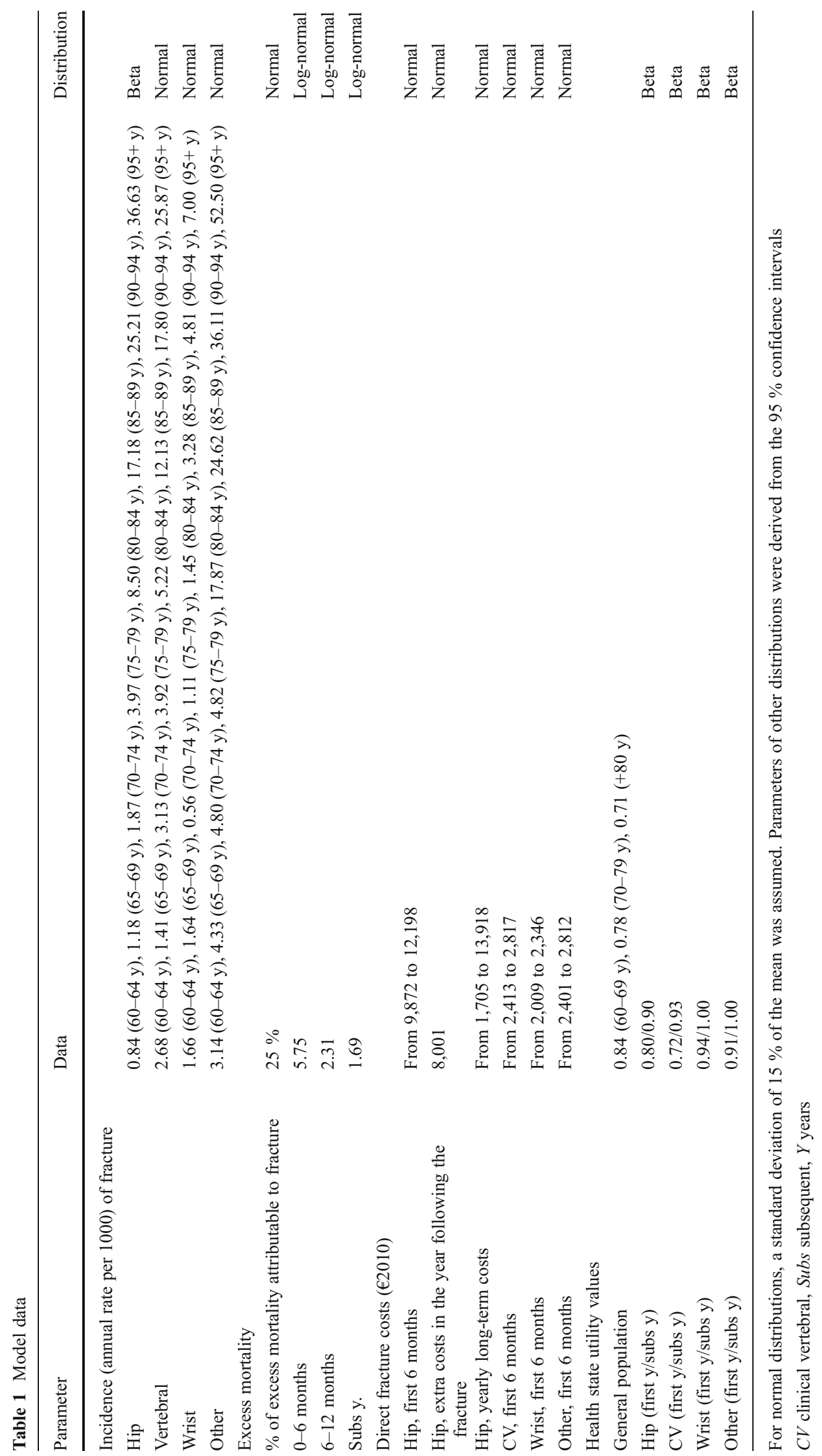


administrated in the first cycle following the fracture, was retrieved from the Belgian national database of hospital bills for the year 2007 [33]. It included the social security cost and the patient out-of-pocket contribution for nursing and residential fees costs only. Extra costs in the year following the hip fracture were derived from the study of Autier et al. [34], which based on a prospective controlled study including 159 women. These costs, estimated at $€ 8,001$ (expressed in $€ 2,010$ ), were equally distributed between the two first cycles following the fracture. Hip fractures are also associated with long-term costs. They were based on the proportion of men being institutionalized following the fracture, ranging from $6 \%$ (for men aged 60 years) to $65 \%$ (for those aged over 90 years) [35]. Because men might be institutionalized later in life, regardless of their hip fracture, an adjustment was made to only include long-term costs attributable to the fracture itself (see Hiligsmann et al. [18] for further explanations). The cost of non-hip fractures has never been estimated in Belgian men and these were quantified relative to hip fracture cost [36]. So, the costs of clinical vertebral, wrist and other fracture represent $17.4 \%, 14.5 \%$ and $17.4 \%$ of the hip fracture cost, respectively. Non-hip fractures were not associated with long-term costs.

Utility values in the general men population as well as relative reductions due to fractures in the year following the fracture and in subsequent years were derived from a systematic review, which suggested reference values for countries that do not have their own database [37]. The reduction of quality-adjusted life-year (QALY) depends on fracture site but also on the number of prior fractures [18]. In the case of an occurrence of a second fracture at the same site, the impact of the first fracture event was reduced by $50 \%$ [18]. For example, if a men with a prior hip fracture suffered another fracture, the relative reduction of utility attributable to the first hip fracture was then 0.95 . For an individual with both a hip and vertebral clinical fracture, the total impact on QALY was assumed to be equal to the sum of the impacts related to each of the fractures. This last assumption is consistent with the study of Tosteson et al. [38], who suggested that the impact of the two fractures is even greater than the sum of the impacts related to each of the fractures. The model, however, does not simulate multiple fractures per 6-monthcycle.

\section{Patient groups}

Analyses were conducted in the population from the MALEO Trial corresponding to men with mean age of 73 years, and with a bone mineral density (BMD) T-score below the threshold value for osteoporosis (i.e., BMD T-score $\leq-2.5$ ) or PVFs at baseline, in order to match the two populations for whom postmenopausal osteoporosis medications are currently reimbursed in Belgium and in most European countries. The
MALEO Trial included in the Full Analysis Set (FAS) 243 men aged 65 to 90 years with osteoporosis as assessed by a mean lumbar spine BMD T-score of -2.7 [15]. The mean BMD at the femoral neck was $0.627\left(\mathrm{~g} / \mathrm{cm}^{2}\right)$, which corresponds to a T-score of approximately -2.2 .

The incidence of fracture in the general population has to be adjusted to accurately reflect the fracture risk in these populations. The relative risks of fracture were calculated from the BMD and the prevalence of vertebral fracture in the target patient groups. The relative risk for BMD was calculated using a method previously described [25]. This method estimates the risk of individuals at a threshold value or below a threshold value in comparison with that in the general population. BMD values at the femoral neck were derived from the National Health and Nutrition Examination Survey (NHANES) III [39] database and 1 standard deviation decrease in BMD was associated with an increase in ageadjusted relative risk of 1.8, 1.4 and 1.6 for clinical vertebral, wrist and other fracture, respectively [40]. For hip fracture, the age-adjusted relative risk ranged from 3.68 at 50 years to 1.93 at 85 years [41]. So, for example, the relative risks of fracture, for men aged 73 years with a BMD of $0.627\left(\mathrm{~g} / \mathrm{cm}^{2}\right)$ at the femoral neck, were estimated at 1.683, 1.529, 1.330 and 1.440 at the hip, clinical vertebral, wrist and other fracture, respectively. The relative risks for men with PVFs were taken from a meta-analysis and were 2.3, 4.4, 1.4 and 1.8 for hip, clinical vertebral, wrist and other fractures, respectively [42]. These relative risks were reduced by $10 \%$ each per decade above the age of 70 years [43]. An increased risk of subsequent fractures was also modelled during the simulation for men who have a prior fracture of the same location, using a previously described method [18].

\section{Strontium ranelate}

The MALEO Trial has been developed in accordance with European guideline on clinical investigation of medicinal products (November 2006). This guideline deals with minimal requirement for marketing indication of a treatment in osteoporosis in men at increased risk fracture once the marketing indication in PMO women has been already granted to the same drug. The MALEO Trial is a controlled study versus placebo on the basis of calcium/vit D supplementation with BMD measure as primary efficacy criteria and a main analysis after 1 year.

In the MALEO Trial [15], a marked increase in the mean lumbar L2-L4 and femoral neck BMD was observed in men with high risk of fractures, similar to that previously observed in women (Table 2). Considering these results and the previously established relationship between change in BMD and reduction in the risk of vertebral and hip fractures with strontium ranelate in women [44, 45], a similar anti-fracture efficacy is expected in men. We therefore assumed, in the base-case 
analysis, the same relative risk reduction of fractures in men as those estimated in women (SOTI and TROPOS trials).

In most cost-effectiveness analyses, efficacy data were retrieved from the entire population of the randomized clinical trials and the modelers charged the full treatment cost. Although, in real-life settings, adherence is far from optimal, this assumption may be incorrect to estimate the potential economic value of a drug and probably underestimates the true underlying risk reduction with therapy since the efficacy from these trials is reduced to some degree because of non-adherence. In the SOTI and TROPOS trials, adherence was collected and per protocol studies including patients with high level of adherence were conducted. The Per Protocol Set strontium (PPS strontium) included all patients from the FAS satisfying a minimum exposure condition based on blood strontium levels criteria. In this analysis, efficacy data from intent-to-treat $[5,7]$ and per-protocol analyses (unpublished data, internal reports SOTI and TROPOS 3-year results) were both tested.

In the base-case analysis, fracture risk reductions were derived from the FAS of the TROPOS and SOTI trials. Strontium ranelate was assumed in this scenario to reduce the risk of hip, wrist and other non-vertebral fractures by $19 \%(\mathrm{RR}=0.81 ; 95 \%$ confidence interval [CI], 0.66-0.98) using the estimated fracture risk reduction for major nonvertebral fractures [7] and the risk of clinical vertebral fracture by $38 \%(\mathrm{RR}=0.62 ; 95 \% \mathrm{CI}, 0.47-0.83)$ [5]. We took a conservative position for the efficacy of strontium ranelate on hip fracture since the results of a post hoc analysis in high-risk women aged over 74 years of age was not incorporated [7]. In the additional scenario, the efficacy of strontium ranelate on non-vertebral fractures was derived from the per-protocol study of the TROPOS Trial including 2,935 osteoporotic women above 70 years of age with high adherence. In this population, strontium ranelate was shown to reduce the risk of hip fracture, as compared to placebo and over 3 years, by $41 \%$ (95\% CI, 5$63 \% ; p=0.025$ ). The risk of any major non-vertebral fractures, used in the model for wrist and other fractures, was reduced by $35 \%(95 \% \mathrm{CI}, 16-49 \% ; p<0.001)$ in the same population. In the per-protocol study conducted in the SOTI trial and including 1,076 women with a mean age of 69 years, the risk of vertebral fracture was reduced by $45 \%$ (95\% CI, 25-57\%; $p<0.001)$.

Patients received treatment in the base-case model for 3 years with the full effect of the treatment during the whole intervention period. After stopping therapy, the effect of strontium ranelate on fracture risk was assumed to decline linearly to zero for a period (called offset time) similar to the duration of therapy in line with a clinical study [46] and prior cost-effectiveness analyses [14]. In a sensitivity analysis, we assessed the impact of poor adherence with strontium ranelate using the same assumption than in prior cost- effectiveness analyses of strontium ranelate in postmenopausal women $[12,13]$. In these analyses, adherence to strontium ranelate was similar to that observed for bisphosphonate therapy in Belgian women [47]. We therefore assumed that $30 \%, 12 \%, 18 \%$ and $15 \%$ of patients discontinued therapy after 3 months, 6 months, 1 year and 2 years, respectively. No treatment effect was assumed for patients who discontinued treatment at 3 months and offset time for non-persistent patients was assumed to be the same as their treatment period. Compliance was estimated at $70.5 \%$ for persistent women [47]. Medication costs and fracture reduction efficacy were assumed to be proportional to compliance.

The annual cost of strontium ranelate was estimated at $€ 477.2$ (Protelos ${ }^{\circledR}, € 109.82$ for a package of 84 sachets) [48], and we assigned the cost of one physician visit $(€ 22.67)$ per year of treatment and the cost of one bone density measurement ( $€ 58.05)$ every secondyear. Adverse events with strontium ranelate are usually mild and transient. In pooled data from the SOTI and TROPOS trials [5, 7], treatment with strontium ranelate, however, was associated with an increase in the annual incidence of VTE, including pulmonary embolism (PE). To account for this in the analysis, VTE was included as a health state in the model during treatment with strontium ranelate. The annual absolute risk of VTE with strontium ranelate was estimated at $0.31 \%$ in women $[5,7]$. In the model, VTE was assumed to be associated with a $10 \%$ utility loss the first year after the event and any utility loss in the second or following years after the event, in agreement with previous health economic publications [49, 50]. The survival rate after PE was estimated at $81.6 \%$ in the clinical trials [5, 7]. Using Belgian estimates of resource utilization based on panel experts [51], the cost of VTE was estimated at $€ 2,622$.

\section{Simulation and analyses}

Microsimulations were performed to estimate the costeffectiveness of strontium ranelate. Each model was run ten times with 200,000 trials (patients) to guarantee the stability of the results and enable variability analyses [23]. For each analysis, the incremental cost-effectiveness ratio (ICER) was computed as the difference between strontium ranelate and no treatment in terms of total costs (expressed in $€ 2,010$ ) divided by the difference between them in terms of effectiveness, expressed in accumulated QALYs. It represents the cost of strontium ranelate (compared with no treatment) per one QALY gained. In Belgium, as in many other countries, no threshold values for ICERs have been defined [52]. Commonly accepted thresholds for costeffectiveness are in the range of $€ 50,000$ [11].

Uncertainty related to model parameters and assumptions was investigated using deterministic and probabilistic 
Table 2 Between treatment comparison of the percentage change in lumbar spine and femoral neck BMD to month 12 relative to baseline in male patients from MALEO and in postmenopausal women in SOTI-TROPOS studies

\begin{tabular}{|c|c|c|c|c|}
\hline \multirow[t]{2}{*}{ Relative change from baseline to $\mathrm{M} 12$} & \multicolumn{2}{|c|}{ Men with osteoporosis } & \multicolumn{2}{|l|}{ PMO women } \\
\hline & & MALEO $N=261(15)$ & TROPOS $N=5,091(7)$ & SOTI $N=1,649(5)$ \\
\hline \multirow[t]{4}{*}{ Lumbar spine BMD } & $N$ & 197 & 3807 & 1361 \\
\hline & $E(\mathrm{SE})$ & $6.2(0.8) \%$ & $7.0(0.2) \%$ & $7.2(0.4) \%$ \\
\hline & $95 \% \mathrm{CI}$ & {$[4.7-7.8]$} & {$[6.6-7.4]$} & {$[6.5-7.9]$} \\
\hline & $p$ value & $p<0.001$ & $p<0.001$ & $p<0.001$ \\
\hline \multirow[t]{4}{*}{ Femoral neck BMD } & $N$ & 178 & 3,759 & 1,326 \\
\hline & $E(\mathrm{SE})$ & $3.2(0.7) \%$ & $3.6(0.2) \%$ & $3.3(0.2) \%$ \\
\hline & $95 \% \mathrm{CI}$ & {$[1.8-4.6]$} & {$[3.3-3.9]$} & {$[2.8-3.8]$} \\
\hline & $p$ value & $p<0.001$ & $p<0.001$ & $p<0.001$ \\
\hline
\end{tabular}

$N$ number of patients with evaluation at both baseline and M12 visits, $E(S E)$ estimate and standard error of the adjusted mean difference (strontium ranelate vs. placebo), $C I$ confidence interval of the estimate, $P M O$ Post-menopausal osteoporosis

sensitivity analyses. Deterministic sensitivity analyses were performed to evaluate the impact of single parameter variations on the results. The baseline parameters for discount rates, fracture risk, fracture disutility, fracture cost and excess mortality were varied over plausible ranges. Changes in therapy cost, monitoring cost, adverse events, offset time and time horizon were also evaluated.

Probabilistic sensitivity analyses were performed with 200 simulations to analyze the effects of uncertainty in all model parameters simultaneously. Distributions used for key model inputs are provided in Table 1. Log-normal distributions were also assumed for fracture risk reduction with strontium ranelate. Cost-effectiveness acceptability curves were then constructed from the incremental cost and QALYs between alternatives for the 200 simulations. They show the probability that strontium ranelate is costeffective compared with no treatment for a range of decision makers' willingness to pay per QALY.

\section{Results}

The lifetime costs, QALYs and ICERs of strontium ranelate compared with no treatment are presented on Table 3 in men

Table 3 Lifetime costs, QALYs and incremental cost-effectiveness ratio (cost in $€$ per QALY gained) of strontium ranelate versus no treatment according to population and anti-fracture efficacy

\begin{tabular}{|c|c|c|c|}
\hline & \multirow[t]{2}{*}{ No treatment } & \multicolumn{2}{|l|}{ Strontium ranelate } \\
\hline & & ITT & PPS \\
\hline \multicolumn{4}{|c|}{ MALEO trial (i.e., BMD T-score of $-2.2 ; 28.1 \%$ prevalent vertebral fracture) } \\
\hline Costs, $€$ & 6,765 & 7,907 & 7,594 \\
\hline QALYs & 7.2156 & 7.2385 & 7.2504 \\
\hline ICER, €/QALY $95 \%$ CI & & $49,798(48,561-51,035)$ & $25,584(24,138-27,030)$ \\
\hline \multicolumn{4}{|c|}{ BMD T-score $\leq-2.5$ (and no prior fracture) } \\
\hline Costs, $€$ & 8,450 & 9,333 & 8,815 \\
\hline QALYs & 7.1970 & 7.2222 & 7.2396 \\
\hline ICER, €/QALY $95 \%$ CI & & $36,270(34,363-38,177)$ & $8,230(7,672-8,888)$ \\
\hline \multicolumn{4}{|l|}{ Prevalent vertebral fracture } \\
\hline Costs, $€$ & 6,189 & 7,325 & 7,063 \\
\hline QALYs & 7.1805 & 7.2053 & 7.2204 \\
\hline ICER, €/QALY $95 \%$ CI & & $42,359(40,210-44,507)$ & $22,895(21,267-24,522)$ \\
\hline
\end{tabular}

ICER is defined as the difference between strontium ranelate and no treatment in terms of costs divided by the difference between them in terms of QALYs

$B M D$ bone mineral density, $C I$ confidence interval of the estimate, ICER incremental cost-effectiveness ratio, $Q A L Y$ quality-adjusted life-year, ITT intention-to-treat (entire population of the clinical trials), PPS per protocol studies (including only patients with high adherence) 
with similar characteristics than those included in the MALEO Trial. Based on anti-fracture efficacy derived from the entire population of the clinical trials, strontium ranelate compared with no treatment was estimated at $€ 49,798 / \mathrm{QALY}$ gained. This value decreased to $€ 36,270$ and to $€ 42,359$ in men with a BMD T-score $\leq-2.5$ (and no prior fractures) and with PVFs at baseline, respectively. Using anti-fracture efficacy from the per-protocol analyses, the cost per QALY gained of strontium ranelate decreased in all simulated populations and remained below a threshold of $€ 30,000$ per QALY gained.

The results of this study were sensitive to adherence to therapy (Fig. 1). When assuming adherence similar to bisphosphonate's adherence for postmenopausal women, the costs per QALY gained of strontium ranelate versus no treatment were respectively $€ 58,117$, and $€ 75,867$ per QALY gained in men with a BMD T-score $\leq-2.5$ and PVF using the anti-fracture efficacy from the intent-to-treat analysis.

Additional deterministic sensitivity analyses were conducted in men with a BMD T-score $\leq-2.5$ aged 73 years using the anti-fracture efficacy from the intent-to-treat analysis (Fig. 2). They showed the estimated ICERs to be modestly sensitive to changes in fracture disutility and fracture cost and quite sensitive to discount rates and changes in fracture risk or mortality excess. The ICERs decreased by $24 \%$ for lower drug cost $(-15 \%)$ and by $38 \%$ when fracture risk was increased by $25 \%$. The ICER was also reduced when assuming no adverse events or no monitoring cost with strontium ranelate.

The results of the probabilistic sensitivity analyses are presented as cost-effectiveness acceptability curves in Fig. 3. The curves indicate the probability that strontium ranelate is cost-effective as a function of the decision maker's willingness to pay per one QALY gained. At an assumed willingness to pay of $€ 45,000$ per QALY, strontium ranelate was cost-effective in $62.0 \%$ and $93.0 \%$ of the simulations

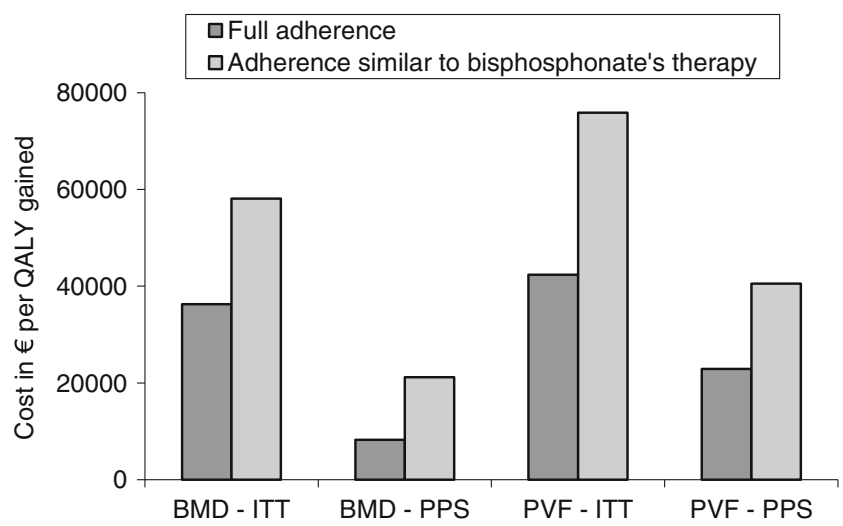

Fig. 1 Potential impact of medication adherence on the cost per QALY gained of strontium ranelate compared with no treatment in men with osteoporosis or prevalent vertebral fracture. $B M D$ bone mineral density $\leq-2.5, I T T$ intention-to-treat, $P P S$ per protocol studies, $P V F$ prevalent vertebral fracture

Base case: $€ 36,270$ per QALY

Fracture risk $+25 \%$

Drug cost $15 \%$ lower

No monitoring cost

Fracture costs $+25 \%$

Offset time 4 years

Discount rates 0\%

No adverse events

Fracture disutility $+25 \%$

Fracture costs $-25 \%$

Fracture disutility $-25 \%$

Discount rates 3\%

Time horizon: 90 years

Drug cost $15 \%$ higher

Offset time 2 years

Discount rates $5 \%$

No mortality excess

Fracture risk $-25 \%$

$$
0
$$

Fig. 2 Tornado diagram for deterministic sensitivity analyses on the cost-effectiveness of strontium ranelate compared with no treatment in men aged 73 years with BMD T-score $\leq-2.5$ using efficacy data from the intent-to-treat analysis

for men aged 73 years with a BMD T-score $\leq-2.5$ aged using efficacy data from the entire population of the clinical trials and from the per-protocol analyses, respectively.

\section{Discussion}

The results of this study suggest that, under the assumption of same relative risk reduction of fractures in men as for women, strontium ranelate is cost-effective compared with no treatment, at commonly accepted thresholds, for men who are similar to patients included in the MALEO Trial.

This study provides the first pharmacoeconomic evaluation of strontium ranelate in male population. Previous

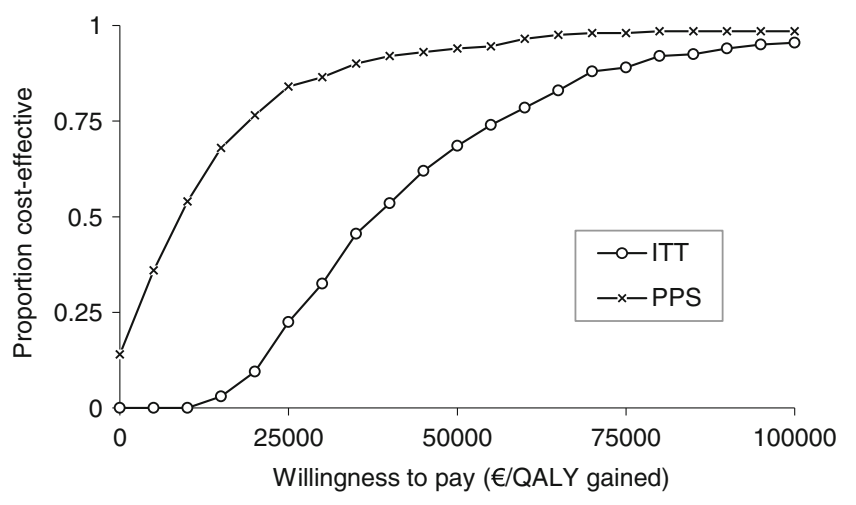

Fig. 3 Cost-effectiveness acceptability curves of strontium ranelate compared with no treatment in men aged 73 years with BMD T-score $\leq-2.5$ according to anti-fracture efficacy. ITT intention-to-treat, PPS per protocol studies 
studies have shown that strontium ranelate was costeffective for post-menopausal women with low BMD [10-14]. Treatment with strontium ranelate was compared with no treatment. Other treatments have been approved for the treatment of male osteoporosis. Data are currently limited on the cost-effectiveness of treating male osteoporosis [53]. In a Swedish setting, treating osteoporotic men with alendronate was shown to be cost-effective versus placebo under the assumption of the same anti-fracture efficacy of alendronate for men as for women [54]. In another analysis, the threshold probability for cost-effective treatment $(\$ 500$ per year, $35 \%$ efficacy) was a 10-year risk of hip fracture that ranged from $2 \%$ at the age of 50 years to $6.5 \%$ at the age of 80 years [55]. Comparison with other drugs could be performed in the future as it was done in women using indirect comparison [12]. These would, however, be much more uncertain than in women because, in addition to uncertainty associated with indirect comparison of efficacy between drugs, there would be uncertainty about the assumption of the same relative risk reduction of fractures in men as for women.

There are some potential limitations to our study that provide uncertainty in the overall results. First, there is no anti-fracture efficacy data of strontium ranelate in the male population. The MALEO Trial was a bridging study and therefore did not represent the gold standard demonstration of anti-fracture efficacy. In accordance with the European guidelines on clinical investigation of medicinal products, the MALEO trial was a controlled study versus placebo with BMD measure as primary efficacy criteria. Similar efficacy data on lumbar spine and femoral neck (FN) BMD between men with osteoporosis at high risk of fracture (MALEO trial [15]) and PMO women (pivotal SOTI, TROPOS trials [5, 7]), however, supports the assumption, in the base-case analysis, of the same relative risk reduction. In addition, the anti-fracture efficacy of strontium ranelate verified in PMO women whatever the baseline characteristics [56] and whatever the 10-year fracture probabilities [57] as well as the relationship between BMD increase and fracture risk reduction $[44,45]$ reinforce this assumption.

Second, even using efficacy data from the entire population of the clinical trials, the cost-effectiveness of the drug in real-life settings could be altered. Many studies have reported that adherence with osteoporosis medications is poor and suboptimal [58], and this may impact on the cost-effectiveness of therapies [21, 59]. A sensitivity analysis assuming adherence similar to bisphosphonate's adherence for postmenopausal women confirms the potential impact of poor adherence on cost-effectiveness. Further research, however, would be required to estimate the costeffectiveness of strontium ranelate in male osteoporosis in real-life settings. This will imply the collection of adherence data with strontium ranelate in male patients as well as on the relationship between poor adherence and fracture risk in men. Additional analyses evaluating the cost-effectiveness of strontium ranelate according to absolute fracture risk would also be valuable. It has been increasingly suggested that treatment should be based on absolute fracture risk rather than on BMD threshold [60]. Although antiosteoporosis treatment are not yet reimbursed based on absolute fracture risk, the development of FRAX $^{\circledR}$ tool, recently available in Belgium [24], would help to identify new high-risk populations of men that could be treated costeffectively by strontium ranelate.

Third, although most of the data were collected from male populations, some of these were derived from studies that were composed mainly of postmenopausal women. So, the impact of fractures on quality of life has not been specifically investigated in populations of men and would require further investigation. The decrease in quality of life due to osteoporotic fractures in men, however, appears comparable to that caused by postmenopausal osteoporotic women [61, 62].

In addition, our analysis was performed from a payer's perspective rather than a societal one, in accordance with Belgian methodological guidelines for pharmacoeconomic evaluations [32]. Non-hip fracture costs were also restricted to acute hospitalization cost but care typically extend beyond this (e.g., drugs, doctors, home care). Taking indirect costs such as productivity losses and other care costs into account would improve the cost-effectiveness of strontium ranelate as sensitivity analysis showed that cost-effectiveness improved with higher fracture costs. Conservative assumption was also used for the costs of vertebral fractures as they were calculated from a relationship between fractures in 1995 [36], and treatment of vertebral fractures has become more expensive in recent years due to an increasing number of surgical procedures [63].

Finally, the generalizability of our results to other settings may be uncertain since the incidence of the disease, the availability of health resources, clinical practice patterns and relative prices may substantially differ between countries and could impact on the costeffectiveness [64]. Cost-effectiveness analysis should ideally be performed in each specific country with local data. However, it is likely that strontium ranelate will also confer cost-effective benefits, compared with no treatment, in countries with similar characteristics than those retained in this analysis.

In conclusion, under the assumption of the same relative risk reduction of fractures in men as for women, this costeffectiveness analysis suggests that strontium ranelate has the potential to be a cost-effective strategy compared with no treatment for men with osteoporosis from a healthcare payer perspective. 
Acknowledgments This work was supported by an unrestricted educational grant from Servier, which had no role in the design or conduct of the study, in the collection, analysis, or interpretation of the data.

Conflicts of interest Mickaël Hiligsmann: research grant, lecture fees and/or consulting fees from Amgen, Pfizer, Novartis, Servier and SMB.

Olivier Bruyère: consulting fees, lecture fees and reimbursement for attending meetings from Servier, GlaxoSmithKline, MSD, Theramex, Galapagos, Rottarpham.

Jean-Yves Reginster: consulting fees or paid advisory boards, Servier, Novartis, Negma, Ely Lilly, Wyeth, Amgen, GlaxoSmithKline, Roche, Merkle, Nycomed, NPS, Theramex; lecture fees when speaking from Merck Sharp and Dohme, Eli Lilly, Rottapharm, IBSA, Genevrier, Novartis, Servier, Roche, GlaxoSmithKline, Teijin, Teva, Ebewee Pharma, Zodiac, Analis, Theramex, Nycomed, Novo-Nordisk; grant support from Bristol Myers Squibb, Merck Sharp \& Dhome, Rottapharm, Teva, Eli Lilly, Novartis, Roche, GlaxoSmithKline, Amgen, and Servier.

Wafa Ben Sedrine has no conflict of interests.

Open Access This article is distributed under the terms of the Creative Commons Attribution Noncommercial License which permits any noncommercial use, distribution, and reproduction in any medium, provided the original author(s) and the source are credited.

\section{References}

1. Ringe JD (2010) Osteoporosis in men. Medicographia 32:71-8

2. Hiligsmann M, Bruyere O, Roberfroid R, et al. (2011) Trends in hip fracture incidence and in the prescription of anti-osteoporosis medications in Belgium (2000-2007). Arthritis Care Res (Hoboken) 2012;64:744-50

3. Kanis JA, Johnell O, Oden A et al (2000) Long-term risk of osteoporotic fracture in Malmo. Osteoporos Int 11:669-74

4. Haentjens P, Magaziner J, Colon-Emeric CS et al (2010) Metaanalysis: excess mortality after hip fracture among older women and men. Ann Intern Med 152:380-90

5. Meunier PJ, Roux C, Seeman E et al (2004) The effects of strontium ranelate on the risk of vertebral fracture in women with postmenopausal osteoporosis. N Engl J Med 350:459-68

6. Reginster JY, Felsenberg D, Boonen S et al (2008) Effects of long-term strontium ranelate treatment on the risk of nonvertebral and vertebral fractures in postmenopausal osteoporosis: results of a five-year, randomized, placebo-controlled trial. Arthritis Rheum 58:1687-95

7. Reginster JY, Seeman E, De Vernejoul MC et al (2005) Strontium ranelate reduces the risk of nonvertebral fractures in postmenopausal women with osteoporosis: Treatment of Peripheral Osteoporosis (TROPOS) study. J Clin Endocrinol Metab 90:2816-22

8. Seeman E, Devogelaer JP, Lorenc R et al (2008) Strontium ranelate reduces the risk of vertebral fractures in patients with osteopenia. $\mathrm{J}$ Bone Miner Res 23:433-8

9. Reginster JY, Kaufman JM, Goemaere S et al (2012) Maintenance of antifracture efficacy over 10 years with strontium ranelate in postmenopausal osteoporosis. Osteoporosis Int 23:1115-22

10. Borgstrom F, Jonsson B, Strom O, Kanis JA (2006) An economic evaluation of strontium ranelate in the treatment of osteoporosis in a Swedish setting: based on the results of the SOTI and TROPOS trials. Osteoporos Int 17:1781-93

11. Borgstrom F, Strom O, Coelho J et al (2010) The cost-effectiveness of strontium ranelate in the UK for the management of osteoporosis. Osteoporos Int 21:339-49

12. Hiligsmann M, Bruyere O, Reginster JY (2010) Cost-effectiveness of strontium ranelate versus risedronate in the treatment of postmenopausal osteoporotic women aged over 75 years. Bone 46:440-6

13. Hiligsmann M, Bruyere O, Reginster JY (2010) Cost-utility of long-term strontium ranelate treatment for postmenopausal osteoporotic women. Osteoporos Int 21:157-65

14. Hiligsmann M, Vanoverberghe M, Neuprez A, Bruyere O, Reginster JY (2010) Cost-effectiveness of strontium ranelate for the prevention and treatment of osteoporosis. Expert Rev Pharmacoecon Outcomes Res 10:359-66

15. Kaufman JM, Audran M, Bianchi G, et al. (2011) Efficacy and safety of strontium ranelate in the treatment of male osteoporosis. Ann Rheum Dis EULAR 11-4358

16. Taylor RS, Drummond MF, Salkeld G, Sullivan SD (2004) Inclusion of cost effectiveness in licensing requirements of new drugs: the fourth hurdle. BMJ 329:972-5

17. Drummond M, Dubois D, Garattini L et al (1999) Current trends in the use of pharmacoeconomics and outcomes research in europe. Value Health 2:323-32

18. Hiligsmann M, Ethgen O, Bruyere O, Richy F, Gathon HJ, Reginster JY (2009) Development and validation of a Markov microsimulation model for the economic evaluation of treatments in osteoporosis. Value Health 12:687-96

19. Hiligsmann M, Gathon HJ, Bruyere O, Ethgen O, Rabenda V, Reginster JY (2010) Cost-effectiveness of osteoporosis screening followed by treatment: the impact of medication adherence. Value Health 13:394-401

20. Hiligsmann M, Rabenda V, Bruyere O, Reginster JY (2010) The clinical and economic burden of non-adherence with oral bisphosphonates in osteoporotic patients. Health Policy 96:170-7

21. Hiligsmann M, Rabenda V, Gathon HJ, Ethgen O, Reginster JY (2010) Potential clinical and economic impact of nonadherence with osteoporosis medications. Calcif Tissue Int 86:202-10

22. Hiligsmann M, Reginster JY (2010) Potential cost-effectiveness of denosumab for the treatment of postmenopausal osteoporotic women. Bone 47:34-40

23. Hiligsmann M, Reginster JY (2011) Cost effectiveness of denosumab compared with oral bisphosphonates in the treatment of postmenopausal osteoporotic women in belgium. PharmacoEconomics 29:895-911

24. Johansson H, Kanis JA, McCloskey EV et al (2011) A FRAX(R) model for the assessment of fracture probability in Belgium. Osteoporos Int 22:453-61

25. Kanis JA, Oden A, Johnell O, Jonsson B, de Laet C, Dawson A (2001) The burden of osteoporotic fractures: a method for setting intervention thresholds. Osteoporos Int 12:417-27

26. Johnell O, Kanis JA, Oden A et al (2004) Mortality after osteoporotic fractures. Osteoporos Int 15:38-42

27. Kanis JA, Oden A, Johnell O, De Laet C, Jonsson B (2004) Excess mortality after hospitalisation for vertebral fracture. Osteoporos Int 15:108-12

28. Kanis JA, Oden A, Johnell O, De Laet C, Jonsson B, Oglesby AK (2003) The components of excess mortality after hip fracture. Bone 32:468-73

29. Cauley JA, Thompson DE, Ensrud KC, Scott JC, Black D (2000) Risk of mortality following clinical fractures. Osteoporos Int 11:556-61

30. Tosteson AN, Jonsson B, Grima DT, O'Brien BJ, Black DM, Adachi JD (2001) Challenges for model-based economic evaluations of postmenopausal osteoporosis interventions. Osteoporos Int 12:849-57

31. Kanis JA, Oden A, Johnell O, De Laet C, Jonsson B (2004) Excess mortality after hospitalisation for vertebral fracture. Osteoporos Int 15:108-12

32. Cleemput I, van Wilder P, Huybrechts M, Vrijens F (2009) Belgian methodological guidelines for pharmacoeconomic evaluations: toward standardization of drug reimbursement requests. Value Health 12:441-49 
33. Hiligsmann M, Gathon HJ, Bruyere O et al (2011) Hospitalisation costs of hip fractures in Belgium. Osteoporos Int 22:332, Abstract, 11th ECCEO-IOF

34. Autier P, Haentjens P, Bentin J et al (2000) Costs induced by hip fractures: a prospective controlled study in Belgium. Belgian Hip Fracture Study Group. Osteoporos Int 11:373-80

35. Reginster JY, Gillet P, Ben Sedrine W et al (1999) Direct costs of hip fractures in patients over 60 years of age in Belgium. PharmacoEconomics 15:507-14

36. Melton LJ 3rd, Gabriel SE, Crowson CS, Tosteson AN, Johnell O, Kanis JA (2003) Cost-equivalence of different osteoporotic fractures. Osteoporos Int 14:383-8

37. Hiligsmann M, Ethgen O, Richy F, Reginster JY (2008) Utility values associated with osteoporotic fracture: a systematic review of the literature. Calcif Tissue Int 82:288-92

38. Tosteson AN, Gabriel SE, Grove MR, Moncur MM, Kneeland TS, Melton LJ 3rd (2001) Impact of hip and vertebral fractures on quality-adjusted life years. Osteoporos Int 12:1042-9

39. Looker AC, Wahner HW, Dunn WL et al (1998) Updated data on proximal femur bone mineral levels of US adults. Osteoporos Int 8:468-89

40. Marshall D, Johnell O, Wedel H (1996) Meta-analysis of how well measures of bone mineral density predict occurrence of osteoporotic fractures. BMJ 312:1254-9

41. Johnell O, Kanis JA, Oden A et al (2005) Predictive value of BMD for hip and other fractures. J Bone Miner Res 20:1185-94

42. Klotzbuecher CM, Ross PD, Landsman PB, Abbott TA 3rd, Berger M (2000) Patients with prior fractures have an increased risk of future fractures: a summary of the literature and statistical synthesis. J Bone Miner Res 15:721-39

43. Kanis JA, Johnell O, De Laet C et al (2004) A meta-analysis of previous fracture and subsequent fracture risk. Bone 35:375-82

44. Bruyere O, Roux C, Badurski J et al (2007) Relationship between change in femoral neck bone mineral density and hip fracture incidence during treatment with strontium ranelate. Curr Med Res Opin 23:3041-5

45. Bruyere O, Roux C, Detilleux J et al (2007) Relationship between bone mineral density changes and fracture risk reduction in patients treated with strontium ranelate. J Clin Endocrinol Metab 92:3076-81

46. Meunier PJ, Roux C, Ortolani S et al (2009) Effects of long-term strontium ranelate treatment on vertebral fracture risk in postmenopausal women with osteoporosis. Osteoporos Int 20:1663-73

47. Rabenda V, Mertens R, Fabri V et al (2008) Adherence to bisphosphonates therapy and hip fracture risk in osteoporotic women. Osteoporos Int 19:811-8

48. Belgian Centre for Pharmacotherapeutic Information, 2011, Available at: http://www.cbip.be/GGR/MPG/MPG_NJ.cfm\#MP 2583 [Accessed 1 July 2011].

49. Borgstrom F, Strom O, Kleman M et al (2011) Cost-effectiveness of bazedoxifene incorporating the $\operatorname{FRAX}(\mathrm{R})$ algorithm in a European perspective. Osteoporos Int 22:955-65
50. Kanis JA, Borgstrom F, Johnell O, Oden A, Sykes D, Jonsson B (2005) Cost-effectiveness of raloxifene in the UK: an economic evaluation based on the MORE study. Osteoporos Int 16:15-25

51. Haentjens P, De Groote K, Annemans L (2004) Prolonged enoxaparin therapy to prevent venous thromboembolism after primary hip or knee replacement. A cost-utility analysis. Arch Orthop Trauma Surg 124:507-17

52. Cleemput I, Neyt M, Thiry N, et al. Valeurs seuils pour le rapport coût-efficacité en soins de santé. Health Technology Assessment (HTA). Bruxelles: Centre fédéral d'expertise des soins de santé (KCE);2008. KCE Reports 100B (D/2008/ 10.273/95). 2008

53. Ebeling PR (2008) Clinical practice. Osteoporosis in men. N Engl J Med 358:1474-82

54. Borgstrom F, Johnell O, Jonsson B, Zethraeus N, Sen SS (2004) Cost effectiveness of alendronate for the treatment of male osteoporosis in Sweden. Bone 34:1064-71

55. Kanis JA, Johnell O, Oden A et al (2005) Intervention thresholds for osteoporosis in men and women: a study based on data from Sweden. Osteoporos Int 16:6-14

56. Roux C, Reginster JY, Fechtenbaum J et al (2006) Vertebral fracture risk reduction with strontium ranelate in women with postmenopausal osteoporosis is independent of baseline risk factors. J Bone Miner Res 21:536-42

57. Kanis JA, Johansson H, Oden A, McCloskey EV (2011) A metaanalysis of the effect of strontium ranelate on the risk of vertebral and non-vertebral fracture in postmenopausal osteoporosis and the interaction with FRAX((R)). Osteoporos Int 22:2347-55

58. Rabenda V, Hiligsmann M, Reginster J-Y (2009) Poor adherence to oral bisphosphonate treatment and its consequences: a review of the evidence. Expert Opin Pharmacother 10:2303-15

59. Kanis JA, Cooper C, Hiligsmann M, Rabenda V, Reginster JY, Rizzoli R (2011) Partial adherence: a new perspective on health economic assessment in osteoporosis. Osteoporos Int 22:2565-73

60. Borgstrom F, Kanis JA (2008) Health economics of osteoporosis. Best Pract Res Clin Endocrinol Metab 22:885-900

61. Adachi JD, Ioannidis G, Pickard L et al (2003) The association between osteoporotic fractures and health-related quality of life as measured by the Health Utilities Index in the Canadian Multicentre Osteoporosis Study (CaMos). Osteoporos Int 14:895-904

62. Papaioannou A, Kennedy CC, Ioannidis G et al (2009) The impact of incident fractures on health-related quality of life: 5 years of data from the Canadian Multicentre Osteoporosis Study. Osteoporos Int 20:703-14

63. Goz V, Koehler SM, Egorova NN et al (2011) Kyphoplasty and vertebroplasty: trends in use in ambulatory and inpatient settings. Spine J 11:737-44

64. Drummond M, Barbieri M, Cook J et al (2009) Transferability of economic evaluations across jurisdictions: ISPOR good research practices task force report. Value Health 12:409-18 